\title{
Locally Non-Equilibrium Magnetoelectric State in Diamagnetic
}

\author{
A.F. KABYCHENKOV* \\ Fryazino Fillial of V.A. Kotel'nikov Institute of Radio Engineering and Electronics of RAS, \\ Vvedensky sq. 1, Fryazino, Moscow region, 141190, Russia
}

(Received September 3, 2017)

\begin{abstract}
In the framework of locally non-equilibrium thermodynamics, the equation of motion of the vector of the magnetic moment in a continuum with spatial dispersion is written. The spectrum of locally non-equilibrium fluctuations of the electromagnetic field and magnetization in a magnetically disordered continuum is determined. It is shown that the dispersion dependences of the fluctuations have energy and impulse gaps. Unstable modes in the diamagnetic state are observed for long relaxation times and weak spatial dispersion. Stationary amplitudes of the modes in the instability region are determined. It is shown that the transition to a locally non-equilibrium state can be regarded as a phase transition. In a new phase, there are damped (normal) and undamped excitations. It is shown that stationary standing modes exist in a limited non-equilibrium medium. The propagation of waves in a locally non-equilibrium continuum with spatial dispersion is considered. It is shown that there are regions of transmission and non-transmission in which there may be frequencies of transparency and opacity.
\end{abstract}

DOI: 10.12693/APhysPolA.134.539

PACS/topics: magnetic moment, spin waves, diamagnetic, instability, phase transition, transparency

\section{Introduction}

The equations of motion for magnetization were written analogously to the equation of motion of a gyroscope $[1,2]$. According to these equations, the magnetic moment vector (with a constant [2] or with a nonconstant value [3]) precesses around an effective magnetic field approaching the equilibrium state. In the spin wave, the precession of the magnetic moment occurs with a phase that varies periodically in space [14]. In Refs. $[5,6]$ the equation of magnetization motion was obtained on the basis of locally non-equilibrium thermodynamics $[7,8]$ by analogy with hydrodynamics [9]. In the last equation, the surface and bulk moments of forces that are not local in time and space are taken into account. On the basis of this equation, locally nonequilibrium waves of magnetization in a disordered dielectric continuum were considered $[5,6]$. In the diamagnetic state, unstable modes were observed in the region of large wavenumbers.

In the present paper, locally non-equilibrium excitations of magnetization are considered with allowance for spatial dispersion [6] and nonlinearity, which limit the region of instability and excitation amplitude.

\section{Equation of movement of the magnetic moment vector in a locally non-equilibrium continuum with spatial dispersion}

The equation of motion of magnetization can be obtained within the framework of non-equilibrium thermo-

*corresponding author; e-mail: kabychenkov@mail.ru dynamics $[7,8]$. The equation of continuity of the magnetization density has the form

$$
\boldsymbol{M}_{, t}+\boldsymbol{R}_{j, j}=\boldsymbol{N},
$$

where (.),,$_{t} \equiv \partial(.) / \partial t$ and $(),.{ }_{j} \equiv \partial(.) / \partial x_{j}$ are the time and space derivatives, $\boldsymbol{R}_{j}=R_{j i} \boldsymbol{e}_{i}, \boldsymbol{e}_{i}$ is the basis vector, $R_{j i}$ is the density of the surface moments of forces, $\boldsymbol{N}$ is the density of the volume moments of forces.

The density of the internal energy of the dielectric satisfies the continuity equation $u,{ }_{t}+\operatorname{div} \boldsymbol{q}=\sigma^{u}$, where $u\left(s, P_{i}, M_{j}\right)$ is the internal energy density, $s$ is the entropy density, $\boldsymbol{q}$ is the internal energy flux density, $\sigma^{u}=$ $\boldsymbol{P},_{t} \boldsymbol{E}+\boldsymbol{M}_{, t} \boldsymbol{B}$ is the source density, $\boldsymbol{P}$ is the electric polarization, $\boldsymbol{E}$ is the electric field, $\boldsymbol{B}$ is the magnetic induction.

On the basis of the continuity equations for the densities of internal energy and magnetization in a dielectric with constant polarization, we can represent the continuity equation for entropy

$$
s,{ }_{t}+J_{j}^{s},{ }_{j}=\sigma,
$$

where $\boldsymbol{J}^{s}$ is the entropy flux. The production of entropy is a positive definite bilinear form

$$
\sigma=\boldsymbol{J}^{n} \boldsymbol{K}^{n}>0
$$

in which the generalized thermodynamic flows and the thermodynamic forces conjugate to them are expressed in the form

$$
\begin{array}{ll}
\boldsymbol{J}^{1}=\boldsymbol{J}^{s}, & \boldsymbol{K}^{1}=(1 / T)_{x}, \\
\boldsymbol{J}^{2}=\boldsymbol{N}, & \boldsymbol{K}^{2}=\boldsymbol{B}^{\text {eff }} / T, \\
\boldsymbol{J}^{3}=R_{j i}, & \boldsymbol{K}^{3}=\boldsymbol{B}_{i j}^{e f f} / T, \\
\boldsymbol{J}^{4}=\boldsymbol{R}, & \boldsymbol{K}^{4}=-2 \boldsymbol{V} / T,
\end{array}
$$

where $T=\partial u / \partial s$ is the temperature, $\boldsymbol{B}^{e f f}=\boldsymbol{B}-\boldsymbol{B}^{m}$ is the effective magnetic induction, $\boldsymbol{B}^{m}=\partial u / \partial \boldsymbol{M}$ is the 
local internal effective magnetic induction, $R_{i j}=R_{[i j]}$ is the symmetric part of the magnetization flux tensor, $B_{i j}^{e f f}$ is the symmetric part of the tensor $B_{i}^{e f f}{ }_{j}, \boldsymbol{R}=$ $-\left(\frac{1}{2}\right) \boldsymbol{e}_{i} e_{i j k} R_{\{j k\}}$ is the vector of the dual antisymmetric part of the magnetization flux tensor $R_{\{j k\}}=-e_{i j k} R_{i}$, $\boldsymbol{V}=\left(\frac{1}{2}\right) \operatorname{rot} \boldsymbol{B}^{e f f}$ is the vortex of effective magnetic induction.

According to the principles of non-equilibrium thermodynamics, the generalized flows and forces are connected by constitutive equations. In the linear approximation, these equations can be represented in the form of an integral relation

$$
\begin{aligned}
& \boldsymbol{K}^{n}(t, \boldsymbol{x})= \\
& \quad \int_{-\infty}^{t} \mathrm{~d} t^{\prime} \int_{-\infty}^{\infty} \mathrm{d}^{3} x^{\prime} I^{n m}\left(t, \boldsymbol{x}, t^{\prime}, \boldsymbol{x}^{\prime}\right) \boldsymbol{J}^{m}\left(t^{\prime}, \boldsymbol{x}^{\prime}\right),
\end{aligned}
$$

where $I^{n m}\left(\boldsymbol{Q}^{(l)}\right)$ are the kinetic coefficients, $\boldsymbol{Q}^{(l)}$ are the parameters, including thermodynamic variables. The constitutive equation takes into account the medium's memory, the causality principle and spatial dispersion. The kinetic coefficients satisfy the symmetric equations [5]. In addition, positivity of the form (3) requires the positivity of the principal minors of the matrix $I^{n m}$ $\left(I^{n n}>0, I^{n n} I^{n+1, n+1}-I^{n, n+1} I^{n+1, n}>0\right.$ and further) $[10]$

Consider highly symmetric continuum (isotropic, from a cubic lattice) at a constant temperature. Then the flows will be unbound. Taking into account the symmetry relations for the kinetic coefficients and expanding the flows in the constitutive equations (5) in a series at the point $(t, \boldsymbol{x})$ and restricting ourselves to the first two terms of the series, we can write the constitutive equations in the form of parabolic (heat conduction) equations

$$
\begin{aligned}
& \tau_{v} \boldsymbol{N},_{t}-\lambda_{v}^{2} \boldsymbol{N},_{n n}+\boldsymbol{N}=\nu \boldsymbol{B}^{e f f}, \\
& \tau_{t} R_{j k, t}-\lambda_{t}^{2} R_{j k},{ }_{n n}+R_{j k}=P_{j k}, \\
& P_{j k}=P_{0 j k}-\delta_{j k}\left(l_{\tau} \tau_{v} R_{n n, t}-l_{\lambda} \lambda_{v}^{2} R_{n n},{ }_{j j}\right), \\
& P_{0 j k}=2 \eta\left(B_{j k}^{e f f}-\delta_{j k} B_{n n}^{e f f} / 3\right)+\delta_{j k} \xi B_{n n}^{e f f}, \\
& \tau_{v} R_{n n, t}-\lambda_{v}^{2} R_{n n}, m m \\
& \tau_{r} \boldsymbol{R},{ }_{t}-\lambda_{n n}^{2} \boldsymbol{R},,_{n n}+\boldsymbol{R}=-2 \chi \boldsymbol{V},
\end{aligned}
$$

where $\tau_{v}$ is the relaxation time of the volume moments of forces, $\tau_{t}, \tau_{1}, \tau_{v}$ and $\tau_{r}$ are the relaxation times of the symmetric and antisymmetric parts of the surface moments of forces, with $\tau_{r}, \tau_{t}$ and $\tau_{1}$ characterizing the relaxation of torsional, shear and longitudinal moments of forces, $\lambda_{v}, \lambda_{t}, \lambda_{r}, \lambda_{1}$ are the characteristic lengths, $\nu$ and $\eta, \xi, \chi$ are the coefficients of the volume and shear, linear, rotational "magnetic viscosity", and from the positive definiteness of the entropy production it follows that all the coefficients are positive, $\tau_{v}=(3 \xi / 2 \eta)\left(\tau_{t}+3 l \tau_{1}\right)$ and $\lambda_{v}^{2}=(3 \xi / 2 \eta)\left(\lambda_{t}^{2}+3 l \lambda_{1}^{2}\right)$ are the relaxation time and spatial dispersion coefficient of the trace of the tensor of the moments of forces, $l_{\tau}=l\left[\left(\tau_{1} / \tau_{v}\right)-1\right], l=I_{12}^{33} / 2 I_{44}^{33}$, $l_{\lambda}=l\left(\left(\lambda_{1}^{2} / \lambda_{v}^{2}\right)-1\right)$
Equations (1) and (6) describe the magnetization dynamics in the linear approximation. Dependence of coefficients on thermodynamic variables and flows (forces) leads to nonlinear terms in the determining equations. In particular, taking into account the dependence of the coefficient of "linear viscosity" on magnetization in the first Eq. (6) leads to the replacement of the body torque

$$
\begin{aligned}
& \nu \boldsymbol{B}^{\text {eff }} \rightarrow g\left[\boldsymbol{M} \boldsymbol{B}^{e f f}\right]+\left(\nu_{11}+\left(\nu_{12}+2 \nu_{44}\right) \boldsymbol{M}^{2}\right) \boldsymbol{B}^{\text {eff }} \\
& -2 \nu_{44}\left[\boldsymbol{M}\left[\boldsymbol{M} \boldsymbol{B}^{e f f}\right]\right] .
\end{aligned}
$$

Consequently, in the highly symmetric locally equilib$\operatorname{rium}\left(\tau_{v}, \tau_{r}, \tau_{r} \rightarrow 0\right)$ without spatial dispersion $\left(\lambda_{v}^{2}, \lambda_{t}^{2}\right.$, $\left.\lambda_{r}^{2} \rightarrow 0\right)$, in the absence of surface moments of forces $(\xi, \chi \rightarrow 0)$, the equation (1) reduces to the usual equation of motion of the vector of the magnetic moment [4].

The system (1), (6) for constant coefficients can be reduced to the equation of motion of the magnetization in the form

$$
\begin{gathered}
\tau_{t} \boldsymbol{M}_{, t t}+\boldsymbol{M},_{t}-\lambda_{t}^{2} \boldsymbol{M},_{t n n}+\eta \Delta \boldsymbol{B}^{e f f} \\
=-\operatorname{grad} \phi^{B}+\boldsymbol{N}^{e}-\operatorname{rot} \boldsymbol{R}^{e},
\end{gathered}
$$

where $\phi^{B}=\eta^{e} B_{j j}^{e f f}+l_{\tau}\left(R_{j j}-\lambda_{v}^{2} R_{j j, n n}\right)$ is the effective scalar potential of the moment of forces, $\eta^{e}=\left[\left(\frac{1}{3}\right) \eta+\right.$ $\left.\xi\left(1-3 l_{\tau}\right)\right]$ is the coefficient of effective magnetic viscosity,

$R_{j j}=$

$\left(3 \xi / \tau_{v}\right) \int_{-\infty}^{t} \mathrm{~d} t^{\prime} \int_{-\infty}^{\infty} \mathrm{d}^{3} x^{\prime} B_{j j}^{e f f}\left(t^{\prime}, x_{n}^{\prime}\right) F_{v}\left(t-t^{\prime}, x_{n}-x_{n}^{\prime}\right)$

is the nonlocal component of the scalar potential, $\boldsymbol{N}^{e}=$ $\boldsymbol{N}+\tau_{t} \boldsymbol{N},_{t}-\lambda_{t}^{2} \boldsymbol{N},_{n n}$ is the effective volumetric moment of forces,

$$
\begin{aligned}
& \boldsymbol{N}(t, \boldsymbol{x})= \\
& \left(\nu / \tau_{v}\right) \int_{-\infty}^{t} \mathrm{~d} t^{\prime} \int_{-\infty}^{\infty} \mathrm{d}^{3} x^{\prime} \boldsymbol{B}^{e f f}\left(t^{\prime}, \boldsymbol{x}^{\prime}\right) F_{v}\left(t-t^{\prime}, x_{n}-x_{n}^{\prime}\right)
\end{aligned}
$$

is the nonlocal volume moment, $\boldsymbol{R}^{e}=\boldsymbol{R}+\tau_{t} \boldsymbol{R}_{,_{t}}-\lambda_{t}^{2} \boldsymbol{R},_{n n}$ is the effective vector potential, the rotor of which gives the moment of forces,

$$
\begin{aligned}
& \boldsymbol{R}(t, \boldsymbol{x})= \\
& \left(-2 \chi / \tau_{r}\right) \int_{-\infty}^{t} \mathrm{~d} t^{\prime} \int_{-\infty}^{\infty} \mathrm{d}^{3} x^{\prime} \boldsymbol{V}\left(t^{\prime}, x_{i}^{\prime}\right) F_{r}\left(t-t^{\prime}, x_{n}-x_{n}^{\prime}\right)
\end{aligned}
$$

is a nonlocal vector potential,

$$
F_{s}=\left(4 \lambda_{s}^{2} \pi\left(t-t^{\prime}\right) / \tau_{s}\right)^{-3 / 2}
$$

$\times \exp \left(-\left(\left(t-t^{\prime}\right) / \tau_{s}\right)-\left(x_{n}^{\prime}-x_{n}\right)^{2} / 4 \lambda_{s}^{2}\left(t-t^{\prime}\right) / \tau_{s}\right]$

are the kernels of integrals, $s=v, v, r$. The non-local components at a given moment of time and at a given point in space are determined by the field values at all previous instants of time at all other points of space.

In the linear approximation, we can write $\boldsymbol{B}^{m}=a \boldsymbol{M}$, where $a$ is the spin-spin interaction constant. In the locally equilibrium state $\left(\tau_{t}, \tau_{v}, \tau_{r} \rightarrow 0\right)$ for weak spatial dispersion $\left(\lambda_{t}, \lambda_{v}, \lambda_{r} \rightarrow 0\right)$, for small inhomogeneity of magnetic induction, Eq. (7) describes diffusion of mag- 
netization. If the inhomogeneity of the magnetization is also small, then it can be seen from (7) that the vector $\boldsymbol{M} \rightarrow \boldsymbol{B} / a$ with the characteristic relaxation time $\tau_{m}=(a \nu)^{-1}$, which is inversely proportional to the coefficient of the body moment of forces and the spinspin interaction constant. In a locally non-equilibrium medium, the parabolic diffusion equation of magnetization is transformed into a hyperbolic equation that admits solutions in the form of transverse and longitudinal waves.

An analogous form (7) has the equations of motion of electric polarization vectors and a vortex electric current.

With a Lorentz gauge and a constant polarization in the dielectric, the magnetic vector potential $(\boldsymbol{B}=\operatorname{rot} \boldsymbol{A})$ satisfies the equation

$$
c^{-2} \boldsymbol{A}, t t-\Delta \boldsymbol{A}=4 \pi \operatorname{rot} \boldsymbol{M} .
$$

The left-hand side of (8) determines transverse electromagnetic waves in a vacuum. The system of Eqs. (7) and (8) describes the coupled waves of magnetization and magnetic vector potential. The fields $\boldsymbol{A}(t, \boldsymbol{r})$ and $\boldsymbol{M}(t, \boldsymbol{r})$ are interrelated by means of the vortices of the fields $\operatorname{rot} \boldsymbol{M}$ and $\operatorname{rot} \boldsymbol{A}$, respectively.

\section{Coupled waves of the magnetic vector potential and magnetization in a locally non-equilibrium continuum with spatial dispersion}

Consider excitations of the continuum in the form of traveling plane waves $\boldsymbol{A}, \boldsymbol{M} \propto \exp (\mathrm{i} \boldsymbol{k} \boldsymbol{x}-\mathrm{i} \omega t)$, where $\boldsymbol{k}$ is the wave vector, $\omega$ is the frequency. Then from (7), (8) follow the relations between the components of the wave

$$
\begin{aligned}
& \left(k^{2}-k_{0}^{2}\right) \boldsymbol{A}=4 \pi \mathrm{i}[\boldsymbol{k} \boldsymbol{M}], \\
& \left(k^{2}-k_{m}^{2}\right) \boldsymbol{M}-c_{l} \boldsymbol{k}(\boldsymbol{k} \boldsymbol{M})=\mathrm{i} k_{c}^{2}[\boldsymbol{k} \boldsymbol{A}],
\end{aligned}
$$

where $\mathrm{k}_{0}=\omega / c, k_{m}^{2}=c_{1} / c_{0}, k_{c}^{2}=c_{2} / c_{0}$ are the characteristic wave numbers, $c_{1}=\mathrm{i} \omega-a\left(\nu / \zeta_{v}\right), c_{2}=$ $\left(\nu / \zeta_{v}\right)+a^{-1} c_{0} k^{2}, c_{0}=a\left(\left(\eta / \zeta_{t}\right)+\left(\chi / \zeta_{r}\right)\right)$ are the components of the wave numbers, $\zeta_{v}=1+\lambda_{v}^{2} k^{2}-\mathrm{i} \omega \tau_{v}$, $\zeta_{t}=1+\lambda_{t}^{2} k^{2}-\mathrm{i} \omega \tau_{t}, \zeta_{r}=1+\lambda_{r}^{2} k^{2}-\mathrm{i} \omega \tau_{r}$ are the dispersion components, $c_{l}=1-\left(a / \zeta_{t} c_{0}\right)\left((4 / 3) \eta+\left(\zeta_{l} / \zeta_{\nu}\right) \xi\right)$ is the dispersion function, $\zeta_{\nu}=1+\lambda_{\nu}^{2} k^{2}-\mathrm{i} \omega \tau_{\nu}, \zeta_{l}=$ $1+\lambda_{\nu}^{2} k^{2}\left(1-3 l_{\lambda}\right)-\mathrm{i} \omega \tau_{\nu}\left(1-3 l_{\tau}\right)$ are the components of the function.

From Eqs. (9) it is clear that unbound longitudinal waves $M$ are described by the dispersion relation $k^{2}=k_{m}^{2} /\left(1-c_{l}\right)$. Transverse waves are coupled waves of vector potential and magnetization with dispersion

$$
\left(q^{2}-\Omega^{2}\right)\left(q^{2}-q_{m}^{2}\right)-4 \pi q_{c}^{2} q^{2}=0,
$$

where $q^{2} \equiv \boldsymbol{q}^{2}=\boldsymbol{k}^{2} \lambda_{m}^{2}, q_{m}^{2},{ }_{c}=k_{m}^{2},{ }_{c} \lambda_{m}^{2}$ are the normalized wave numbers, $\lambda_{m}=\mathrm{c} \tau_{m}$ is the characteristic length, and $\Omega=\omega \tau_{m}$ is the normalized frequency. Because of the isotropy of the medium, the dispersion relation depends only on the square of the wave number. At $4 \pi q_{c}^{2} \rightarrow 0$ the dispersion relation of the coupled waves decays into the dispersion relations of unbound electromagnetic waves $q^{2}=\Omega^{2}$ and magnetization waves $q^{2}=q_{m}^{2}$.
By definition, $\boldsymbol{M}=\left(\chi_{0} /\left(1+4 \pi \chi_{0}\right)\right) \boldsymbol{B}$, where $\chi_{0}$ is the magnetic susceptibility [4]. From (10) it follows that $\chi_{0}=\chi_{00} /\left(1-4 \pi \chi_{00}\right)$, where $\chi_{00}=q_{c}^{2} /\left(q^{2}-q_{m}^{2}\right)$. For $q, \Omega \rightarrow 0$ the quasi-static homogeneous susceptibility $\chi_{0}=1 /(a-4 \pi)$. In paramagnets $\chi_{0}>0$ and, consequently, $a>4 \pi$, in diamagnets $\chi_{0}<0$ and, consequently, $a<4 \pi$.

\section{Spectrum of locally non-equilibrium fluctuations}

We consider the fluctuations of the magnetization in the form of wave packets consisting of plane waves with a complex frequency and a real wave number.

Equation (10) can be represented in the form

$$
\begin{aligned}
& a_{06} \Omega^{6}+a_{04} \Omega^{4}+a_{02} \Omega^{2}+a_{00} \\
& \quad+\mathrm{i} \Omega\left(a_{05} \Omega^{4}+a_{03} \Omega^{2}+a_{01}\right)=0,
\end{aligned}
$$

where $a_{06}=\theta_{v} \theta_{1}, a_{05}=a_{050}+a_{051} q^{2}, a_{050}=\theta_{v} \theta_{0}+\theta_{1}$, $a_{051}=\theta_{v} \theta_{0 \lambda}+\theta_{1} \lambda_{y m}^{2}, a_{04}=a_{040}+a_{041} q^{2}+a_{042} q^{4}$, $a_{040}=-\theta_{v}-\theta_{0}-\theta_{1}, a_{041}=-a_{06}\left(1+a \gamma_{0}\right)-\theta_{0 \lambda}-$ $\theta_{0} \lambda_{y m}^{2}-\theta_{v} \lambda_{0}^{2}, a_{042}=-\theta_{0 \lambda} \lambda_{y m}^{2}-\theta_{v} \lambda_{t m}^{2} \lambda_{r m}^{2}, a_{03}=$ $a_{030}+a_{031} q^{2}+a_{032} q^{4}+a_{033} q^{6}, a_{030}=-1-\theta_{0}, a_{031}=$ $-a_{050}-a \gamma_{01}-\theta_{0 \lambda}-\lambda_{1}^{2}, a_{032}=-a_{051}-a \gamma_{01 \lambda}-\lambda_{2}^{2}, a_{033}=$ $-\lambda_{y m}^{2} \lambda_{t m}^{2} \lambda_{r m}^{2}, a_{02}=a_{020}+a_{021} q^{2}+a_{022} q^{4}+a_{023} q^{6}$, $a_{020}=1, a_{021}=\theta_{v}+\theta_{0}+\alpha \theta_{1}+a \gamma_{1}+\lambda_{0}^{2}, a_{022}=$ $a\left(a_{06} \alpha \gamma_{0}+\gamma_{1} \lambda_{y m}^{2}+\gamma_{1 \lambda}\right)+\theta_{0} \lambda_{y m}^{2}+\theta_{v} \lambda_{0}^{2}+\theta_{0 \lambda}+\lambda_{t m}^{2} \lambda_{r m}^{2}$, $a_{023}=-a_{042}+a \gamma_{1 \lambda} \lambda_{y m}^{2}, a_{01}=a_{011} q^{2}+a_{012} q^{4}+a_{013} q^{6}+$ $a_{014} q^{8}, a_{011}=1+\alpha \theta_{0}, a_{012}=\alpha\left(a \gamma_{01}+\theta_{0 \lambda}\right)+\lambda_{1}^{2}$, $a_{013}=\alpha a \gamma_{01 \lambda}+\lambda_{2}^{2}, a_{014}=-a_{033}, a_{00}=a_{001} q^{2}+a_{002} q^{4}+$ $a_{003} q^{6}+a_{004} q^{8}, a_{001}=-\alpha, a_{002}=-\alpha\left(a \gamma_{1}+\lambda_{0}^{2}\right), a_{003}=$ $-\alpha\left[a\left(\gamma_{1 \lambda}+\gamma_{1} \lambda_{y m}^{2}\right)+\lambda_{t m}^{2} \lambda_{r m}^{2}\right], a_{004}=-\alpha a \gamma_{1 \lambda} \lambda_{y m}^{2}$ are the coefficients, $\theta_{v}=\tau_{v} / \tau_{m}, \theta_{t}=\tau_{t} / \tau_{m}, \theta_{r}=\tau_{r} / \tau_{m}$ are the normalized relaxation times, $\theta_{1}=\theta_{t} \theta_{r}, \theta_{0}=\theta_{t}+\theta_{r}$-are the characteristic relaxation times, $\theta_{0 \lambda}=\theta_{t} \lambda_{r m}^{2}+\theta_{r} \lambda_{t m}^{2}$, $\lambda_{y m}^{2}=\lambda_{v}^{2} / \lambda_{m}^{2}, \lambda_{t m}^{2}=\lambda_{t}^{2} / \lambda_{m}^{2}, \lambda_{r m}^{2}=\lambda_{r}^{2} / \lambda_{m}^{2}$ are the normalized coefficients of spatial dispersion, $\lambda_{0}^{2}=\lambda_{t m}^{2}+\lambda_{r m}^{2}$, $\lambda_{1}^{2}=\lambda_{0}^{2}+\lambda_{y m}^{2}, \lambda_{2}^{2}=\lambda_{y m}^{2} \lambda_{0}^{2}+\lambda_{t m}^{2} \lambda_{r m}^{2}, \gamma_{\eta}=v_{\eta}^{2} / c^{2}, \gamma_{\chi}=$ $v_{\chi}^{2} / c^{2}$ are the ratios of characteristic magnetization waves $v_{\eta}^{2}=\eta / \tau_{t}, v_{\chi}^{2}=\chi / \tau_{r}$ and speed of light, $\gamma_{0}=\gamma_{\eta}+\gamma_{\chi}$, $\gamma_{1}=\gamma_{\eta} \theta_{t}+\gamma_{\chi} \theta_{r}, \gamma_{01}=\gamma_{0} \theta_{1}+\gamma_{1} \theta_{v}$ are the characteristic ratios of velocities, $\gamma_{1 \lambda}=\gamma_{\eta} \theta_{t} \lambda_{r m}^{2}+\gamma_{\chi} \theta_{r} \lambda_{t m}^{2}$, $\gamma_{01 \lambda}=\gamma_{0} \theta_{1} \lambda_{y m}^{2}+\gamma_{1 \lambda} \theta_{v}$ are the renormalized by the spatial dispersion the ratio of velocities, $\alpha=1-(4 \pi / a)$ is the ratio of the reciprocal susceptibility and the spin-spin interaction constant.

Separating the real and imaginary parts from (11), we obtain a system of equations

$$
\begin{aligned}
& a_{3}^{r} \Omega^{\prime 6}+a_{2}^{r} \Omega^{\prime 4}+a_{1}^{r} \Omega^{\prime 2}+a_{0}^{r}=0, \\
& a_{2}^{m} \Omega^{\prime 4}+a_{1}^{m} \Omega^{\prime 2}+a_{0}^{m}=0,
\end{aligned}
$$

where $a_{3}^{r}=a_{06}, a_{2}^{r}=a_{04}-5 a_{05} \Omega^{\prime \prime}-15 a_{06} \Omega^{\prime \prime 2}, a_{1}^{r}=$ $a_{02}-3 a_{03} \Omega^{\prime \prime}-6 a_{04} \Omega^{\prime \prime 2}+10 a_{05} \Omega^{\prime \prime 3}+15 a_{06} \Omega^{\prime \prime 4}, a_{0}^{r}=a_{00^{-}}$ $a_{01} \Omega^{\prime \prime}-a_{02} \Omega^{\prime \prime 2}+a_{03} \Omega^{\prime \prime 3}+a_{04} \Omega^{\prime \prime 4}-a_{05} \Omega^{\prime \prime 5}-a_{06} \Omega^{\prime \prime 6}$ are the coefficients in the first equation, $a_{2}^{m}=a_{05}+6 a_{06} \Omega^{\prime \prime}$, $a_{1}^{m}=a_{03}+4 a_{04} \Omega^{\prime \prime}-10 a_{05} \Omega^{\prime \prime 2}-20 a_{06} \Omega^{\prime \prime 3}, a_{0}^{m}=a_{01}+$ 
$2 a_{02} \Omega^{\prime \prime}-3 a_{03} \Omega^{\prime \prime 2}-4 a_{04} \Omega^{\prime \prime 3}+5 a_{05} \Omega^{\prime \prime 4}+6 a_{06} \Omega^{\prime \prime 5}$ are the coefficients in the second equation.

The second Eq. (12) with the condition $a_{2}^{m} \neq 0$ gives the dispersion relations of the spin-electromagnetic (SE) and electromagnetic-spin (ES) modes in an implicit form $\Omega_{1}^{\prime 2},{ }_{2}=-\left(a_{1}^{m} / 2 a_{2}^{m}\right) \pm\left[\left(a_{1}^{m} / 2 a_{2}^{m}\right)^{2}-\left(a_{0}^{m} / a_{2}^{m}\right)\right]^{\frac{1}{2}}$, in which the imaginary part of the frequency is determined from equation

$$
i_{0} I_{0}+i_{1} I_{1}+\left(a_{3}^{r}\right)^{2} I_{2}=0
$$

where $i_{0}=a_{0}^{r} a_{1}^{m}-a_{1}^{r} a_{0}^{m}, I_{0}=a_{2}^{m}\left(2 a_{3}^{r} a_{0}^{m}+a_{2}^{r} a_{1}^{m}-\right.$ $\left.a_{1}^{r} a_{2}^{m}\right)-a_{3}^{r}\left(a_{1}^{m}\right)^{2}, i_{1}=a_{0}^{r} a_{2}^{m}-a_{2}^{r} a_{0}^{m}, I_{1}=a_{2}^{m} i_{1}+a_{3}^{r} a_{0}^{m} a_{1}^{m}$, $I_{2}=\left(a_{0}^{m}\right)^{3}$ are the functions of the coefficients. Substituting the expressions for the coefficients from (12) into (14), we obtain the equation of the fifteenth degree for the imaginary part of the frequency

$$
\Sigma_{n=0}^{n=15} e_{n} \Omega^{\prime \prime n}=0,
$$

where $e_{n}=\Sigma_{m, l}^{m+l=n} i_{0 m} I_{0 l}+\sum_{p, r}^{p+r=n} i_{1 p} I_{l r}+a_{06}^{2} I_{2 n}$ are coefficients dependent on $q^{2}, 0 \leq m \leq 9,0 \leq$ $l \leq 6,0 \leq p \leq 7,0 \leq r \leq 8$ are integers, $i_{00}=a_{03} a_{00}-a_{02} a_{01}, i_{01}=4 a_{04} a_{00}+2 a_{03} a_{01}-2 a_{02}^{2}$, $i_{02}=-10 a_{05} a_{00}+2 a_{04} a_{01}+8 a_{03} a_{02}, i_{03}=-20 a_{06} a_{00}+$ $12 a_{04} a_{02}-8 a_{03}^{2}, i_{04}=5 a_{06} a_{01}-15 a_{05} a_{02}-25 a_{04} a_{03}$, $i_{05}=-16 a_{06} a_{02}+34 a_{05} a_{03}-20 a_{04}^{2}, i_{06}=42 a_{06} a_{03}+$ $56 a_{05} a_{04}, i_{07}=72 a_{06} a_{04}-40 a_{05}^{2}, i_{08}=-105 a_{06} a_{05}$, $i_{09}=-70 a_{06}^{2}$ are the coefficients of $\Omega^{\prime \prime}$ in the function $i_{0}, I_{00}=a_{06}\left(2 a_{05} a_{01}-a_{03}^{2}\right)+a_{05}\left(a_{04} a_{03}-a_{05} a_{02}\right), I_{01}=$ $a_{06}\left(12 a_{06} a_{01}-8 a_{05} a_{02}-2 a_{04} a_{03}\right)+a_{05}\left(4 a_{04}^{2}-2 a_{05} a_{03}\right)$, $I_{02}=a_{06}\left(-12 a_{06} a_{02}+5 a_{05} a_{03}+8 a_{04}^{2}\right)-24 a_{05}^{2} a_{04}, I_{03}=$ $a_{06}\left(22 a_{06} a_{03}-116 a_{05} a_{04}\right)+40 a_{05}^{3}, I_{04}=a_{06}\left(325 a_{05}^{2}-\right.$ $\left.152 a_{06} a_{04}\right), I_{05}=932 a_{06}^{2} a_{05}, I_{06}=932 a_{06}^{3}$ are the coefficients of $\Omega^{\prime \prime}$ in the function $I_{0}, i_{10}=a_{05} a_{00}-a_{04} a_{01}$, $i_{11}=6 a_{06} a_{00}+4 a_{05} a_{01}-2 a_{04} a_{02}, i_{12}=9 a_{06} a_{01}+$ $9 a_{05} a_{02}+3 a_{04} a_{03}, i_{13}=24 a_{06} a_{02}-14 a_{05} a_{03}+4 a_{04}^{2}, i_{14}=$ $-39 a_{06} a_{03}-24 a_{05} a_{04}, i_{15}=-60 a_{06} a_{04}+24 a_{05}^{2}, i_{16}=$ $98 a_{06} a_{05}, i_{17}=84 a_{06}^{2}$ are the coefficients of $\Omega^{\prime \prime}$ in the function $i_{1}, I_{10}=a_{06} a_{03} a_{01}+a_{05}\left(a_{05} a_{00}-a_{04} a_{01}\right), I_{11}=$ $a_{06}\left(12 a_{05} a_{00}-2 a_{04} a_{01}+2 a_{03} a_{02}\right)+a_{05}\left(4 a_{05} a_{01}-2 a_{04} a_{02}\right)$, $I_{12}=a_{06}\left(36 a_{06} a_{00}+23 a_{05} a_{01}-4 a_{04} a_{02}-3 a_{03}^{2}\right)+$ $a_{05}\left(9 a_{05} a_{02}+3 a_{04} a_{03}\right), I_{13}=a_{06}\left(34 a_{06} a_{01}+58 a_{05} a_{02}+\right.$ $\left.2 a_{04} a_{03}\right)+a_{05}\left(4 a_{04}^{2}-14 a_{05} a_{03}\right), I_{14}=a_{06}\left(104 a_{06} a_{02}-\right.$ $\left.88 a_{05} a_{03}+8 a_{04}^{2}\right)-24 a_{05}^{2} a_{04}, I_{15}=-a_{06}\left(-168 a_{06} a_{03}-\right.$ $\left.144 a_{05} a_{04}\right)+24 a_{05}^{3}, \quad I_{16}=a_{06}\left(-256 a_{06} a_{04}+192 a_{05}^{2}\right)$, $I_{17}=512 a_{06}^{2} a_{05}, I_{18}=384 a_{06}^{3}$ are the coefficients of $\Omega^{\prime \prime}$ in the function $I_{1}, I_{20}=a_{01}^{3}, I_{21}=6 a_{01}^{2} a_{02}$, $I_{22}=a_{01}\left(-9 a_{01} a_{03}+12 a_{02}^{2}\right), I_{23}=-12 a_{01}\left(a_{01} a_{04}+\right.$ $\left.3 a_{02} a_{03}\right)+8 a_{02}^{3}, \quad I_{24}=a_{01}\left(15 a_{01} a_{05}-48 a_{02} a_{04}+\right.$ $\left.27 a_{03}^{2}\right)-36 a_{02}^{2} a_{03}, \quad I_{25}=a_{01}\left(18 a_{01} a_{06}+60 a_{02} a_{05}+\right.$ $\left.72 a_{03} a_{04}\right)+a_{02}\left(-48 a_{02} a_{04}+54 a_{03}^{2}\right), I_{26}=a_{01}\left(72 a_{02} a_{06}-\right.$ $\left.90 a_{03} a_{05}+48 a_{04}^{2}\right)+a_{02}\left(60 a_{02} a_{05}+144 a_{03} a_{04}\right)-27 a_{03}^{3}$, $I_{27}=a_{01}\left(-108 a_{03} a_{06}-120 a_{04} a_{05}\right)+a_{02}\left(72 a_{02} a_{06}-\right.$ $\left.180 a_{03} a_{05}+96 a_{04}^{2}\right)-108 a_{03}^{2} a_{04}, I_{28}=a_{01}\left(-144 a_{04} a_{06}+\right.$ $\left.75 a_{05}^{2}\right)+a_{02}\left(-216 a_{03} a_{06}-240 a_{04} a_{05}\right)+a_{03} 144\left(a_{03} a_{05}-\right.$ $\left.a_{04}^{2}\right), I_{29}=a_{01} 180 a_{05} a_{06}+a_{02}\left(-288 a_{04} a_{06}+150 a_{05}^{2}\right)+$ $a_{03}\left(162 a_{03} a_{06}+360 a_{04} a_{05}\right)-64 a_{04}^{3}, I_{210}=a_{01} 108 a_{06}^{2}+$ $360 a_{02} a_{05} a_{06}+a_{03}\left(432 a_{04} a_{06}-225 a_{05}^{2}\right)+240 a_{04}^{2} a_{05}$, $I_{211}=216 a_{02} a_{06}^{2}-540 a_{03} a_{05} a_{06}+a_{04}\left(288 a_{04} a_{06}-\right.$ $\left.300 a_{05}^{2}\right), I_{212}=-324 a_{03} a_{06}^{2}-720 a_{04} a_{05} a_{06}+125 a_{05}^{3}$, $I_{213}=-432 a_{04} a_{06}^{2}+450 a_{05}^{2} a_{06}, I_{214}=540 a_{05} a_{06}^{2}, I_{215}=$ $216 a_{06}^{3}$ are the coefficients of $\Omega^{\prime \prime}$ in the function $I_{2}$.

In the general case, Eq. (15) can have up to fifteen solutions $\Omega_{n}^{\prime \prime}\left(q^{2}\right)$. Each solution yields up to two branches of the spectrum (13) $\Omega_{1}^{\prime 2},{ }_{2}\left(\Omega_{n}^{\prime \prime}, q^{2}\right)$. Dissipation splits the spectrum. The substitution of (13) in (11) gives the relation between the amplitudes in the mode. A large number of dispersion branches leads to complex nonequilibrium motion of the magnetization.

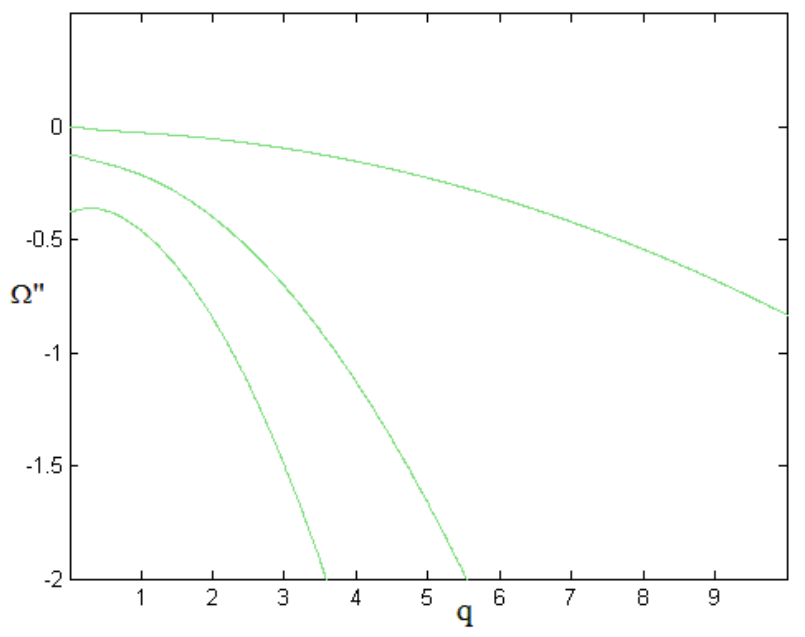

Fig. 1. Dependence of the imaginary part of the frequency on the wave number for paramagnets.

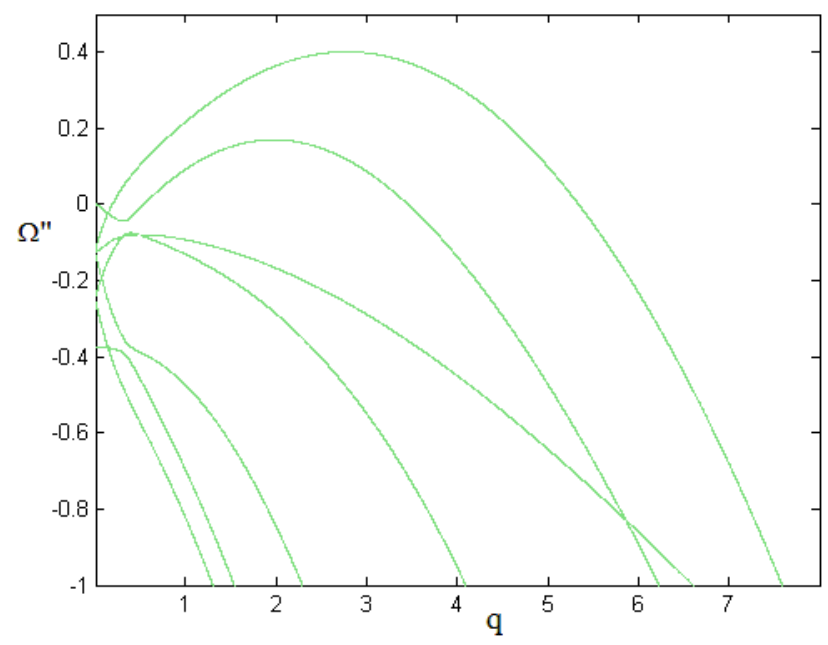

Fig. 2. Dependence of the imaginary part of the frequency on the wave number for diamagnetics.

At the point $q=0$, along with the zero solution, there exist nonzero solutions $(15) \Omega_{n}^{\prime \prime}(q=0) \equiv \Omega_{n 0}^{\prime \prime}$ satisfying the equation of the fourteenth degree $\Sigma_{n=1}^{n=15} e_{n 0} \Omega_{0}^{\prime \prime n-1}=$ 0, where $e_{n 0}=e_{n}(q=0)$ (Figs. 1, 2). The frequencies of homogeneous states are determined by (13) for $q=0$ and $\Omega^{\prime \prime}=\Omega_{n 0}^{\prime \prime}$. In particular, the frequencies of undamped homogeneous oscillations are $\Omega_{20}^{\prime}=\left[\left(1+\theta_{0}\right) /\left(\theta_{v} \theta_{0}+\theta_{1}\right)\right]^{\frac{1}{2}}$ 


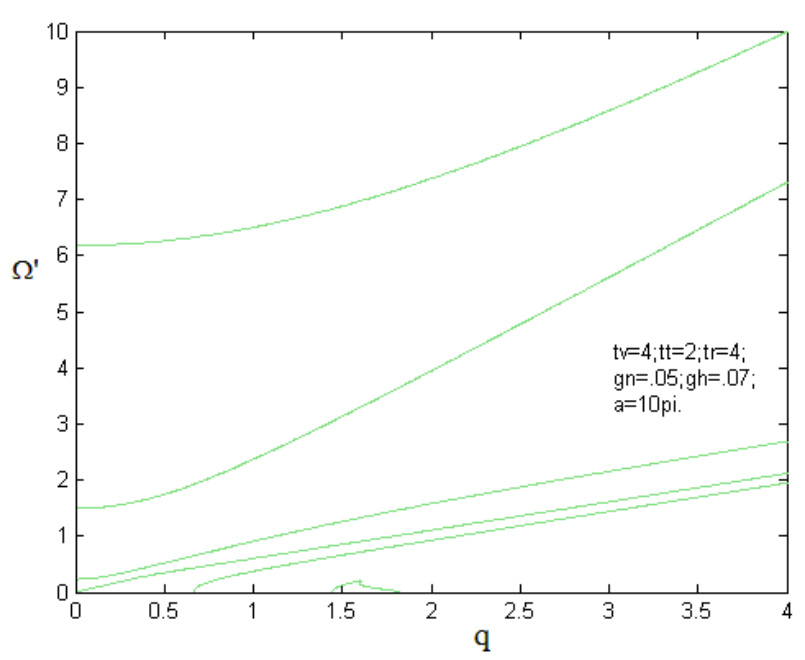

Fig. 3. Dependence of the real part of the frequency on the wave number for paramagnets.

and $\Omega_{10}^{\prime}=0$. ES branches have energy gaps (Fig. 3). The gap $\Omega_{20}^{\prime}$ is determined by the relaxation times. SE branches have impulse clearances (threshold) $q_{t}$, which are satisfied by the condition $\Omega^{\prime}\left(q_{t}\right)=0$. The system (12) gives the equations $a_{0}^{r}\left(q_{t}\right)=0, a_{0}^{m}\left(q_{t}\right)=0$, from which it is seen that the branch with $\Omega^{\prime \prime}\left(q_{t}\right)=0$ does not have the threshold $q_{t 0}=0$ and branches with nonzero $\Omega^{\prime \prime}\left(q_{t}\right)$ have a threshold (Fig. 3). In particular, in the linear approximation with respect to $\Omega^{\prime \prime}$ and $q^{2}$, the threshold $q_{t 1}^{2}=2 \alpha /\left(1+\alpha^{2}\left(\theta_{t}^{2}+\theta_{r}^{2}\right)-2 \alpha\left(\theta_{v}+a \gamma_{1}+\lambda_{0}^{2}\right)\right)>0$ in the paramagnet and the threshold is absent in the diamagnet. The gaps are due to the dynamic interaction of the electromagnetic and spin non-equilibrium subsystems.

There are points of stability loss $q_{c}$ for which $\Omega^{\prime \prime}\left(q_{c}\right)=$ 0 . At the point $q_{c}$, the imaginary part of $\Omega$ changes sign. The amplitudes of the excitations with $\Omega^{\prime \prime}<0$ and $\Omega^{\prime \prime}>0$ decrease and increase with time, respectively. The critical wave numbers $q_{c}$ satisfy the equation $\Sigma_{n=1}^{n=13} e_{0 n} q_{c}^{2 n}=0$. The equation has a zero solution $q_{c}=0$. In paramagnets, the equation has no non-zero solutions since all the coefficients of the same sign. In diamagnetics, solutions exist for weak spatial dispersion and long relaxation times. At the points $q_{c}$, along with the zero solution, there exist solutions satisfying the equation $\sum_{n=1}^{n=15} e_{n c} \Omega_{c}^{\prime \prime n-1}=0$, where $e_{n c}=e_{n}\left(q_{c}\right)$.

In the particular case, when $\theta_{v}=\theta_{r}=0, \lambda_{y m}^{2}=\lambda_{r m}^{2}=$ 0 and therefore $a_{3}^{r}=a_{2}^{m}=0$ and $\theta_{t} \neq 0, \lambda_{t m}^{2} \neq 0$, the system (12) gives the dispersion relation

$$
\begin{aligned}
& \Omega^{\prime 2}=\left[\left(a_{q}+\alpha \theta_{t}\right) q^{2}+2 a_{q \eta} \Omega^{\prime \prime}+3 a_{q t} \Omega^{\prime \prime 2}\right. \\
& \left.\quad+4 \theta_{t} \Omega^{\prime \prime 3}\right] /\left(a_{q t}+4 \theta_{t} \Omega^{\prime \prime}\right),
\end{aligned}
$$

where $a_{q}=1+\lambda_{t m}^{2} q^{2}, a_{q \eta}=a_{q}+\theta_{t}\left(1+a \gamma_{\eta}\right) q^{2}$, $a_{q t}=a_{q}+\theta_{t}$ and equation

$$
\sum_{n=0}^{n=6} e_{n}^{(t)} \Omega^{\prime \prime n}=0,
$$

where $e_{6}^{(t)}=64 \theta_{t}^{3}, e_{5}^{(t)}=96 \theta_{t}^{2} a_{q t}, e_{4}^{(t)}=16 \theta_{t}\left(2 \theta_{t} a_{q \eta}+\right.$ $\left.3 a_{q t}^{2}\right), e_{3}^{(t)}=8 a_{q t}\left(4 \theta_{t} a_{q \eta}+a_{q t}^{2}\right), \quad e_{2}^{(t)}=8 a_{q t}^{2} a_{q \eta}+$ $\left(2 \theta_{t} / a_{q t}^{2}\right) e_{1}^{(t)}, e_{1}^{(t)}=2 a_{q t}\left[a_{q t}\left(a_{q}+\alpha \theta_{t}\right) q^{2}+\left(a_{q}+\theta_{t}\left(a \gamma_{\eta}-\right.\right.\right.$ 1) $\left.\left.q^{2}\right)^{2}+4 \theta_{t}(1-\alpha)\left(a_{q}+\theta_{t} a \gamma_{\eta} q^{2}\right)\right], e_{0}^{(t)}=\left[a_{q} a_{q t}\left(a_{q}+\right.\right.$ $\left.\left.\left.\theta_{t} a \gamma_{\eta}\right) q^{2}\right)+\theta_{t}^{2}\left(a_{q}+\alpha \theta_{t}\right) q^{2}\right](1-\alpha) q^{2}$. If $\alpha>0$, then all the coefficients $e_{n}^{(t)}>0$, therefore the solution (15') $\Omega_{n}^{\prime \prime}<0$ and, consequently, the paramagnetic state will be stable. The diamagnetic state $(\alpha<0)$ can be unstable due to the coefficient $\left(a_{q}+\alpha \theta_{t}\right)$. It can be seen that the spatial dispersion suppresses the instability in the region of large $q^{2}$. At weak attenuation (in particular, near $q_{c}$ ), the wave velocity will be larger in a paramagnet than in a diamagnet. In the particular case, when $\theta_{v}=\theta_{t}=0, \lambda_{y m}^{2}=\lambda_{t m}^{2}=0$ and $\theta_{r} \neq 0, \lambda_{r m}^{2} \neq 0$, the results are obtained from the previous ones by replacing the indices " $t$ " by " $r$ " and " $~ \eta$ " by " $\chi$ ". In the special case, when $\theta_{t}=\theta_{r}=0, \lambda_{t m}^{2}=\lambda_{r m}^{2}=0$ and $\theta_{v} \neq 0$, $\lambda_{y m}^{2} \neq 0$, the paramagnetic and diamagnetic states will be stable. Consequently, instability is caused by nonequilibrium surface moments of forces.

The instability can be explained on the basis of the negative energy of the waves [11-14]. SE waves excite slow and fast ES waves. If the phase velocities of the SE waves are greater than the velocity of the ES waves in the continuum, then slow ES waves will be unstable [11, 12]. As a result, the amplitudes of the ES and SE of the wave increase (an analog of the anomalous Doppler effect) $[13,14]$. This means that the energy of the continuum with waves is less than the energy of the continuum without waves.

Let us consider numerical solutions. Figure 1 shows the dependence of $\Omega^{\prime \prime}(q)$ obtained in (15) in the paramagnet for the parameters $\theta_{v}=4, \theta_{t}=2, \theta_{r}=4, \gamma_{\eta}=0.05$, $\gamma_{\chi}=0.07, \lambda_{y m}^{2}=\lambda_{t m}^{2}=\lambda_{r m}^{2}=0.4, a=10 \pi$. It can be seen that there exists a set of homogeneous oscillations with increasing damping, in addition to the zero undamped mode. In the region of large $q$, the branch $\Omega_{1}^{\prime \prime}$ tends to a constant and the branches $\Omega_{0}^{\prime \prime}$ and $\Omega_{2}^{\prime \prime}$ are determined to be close to quadratic functions. Figure 3 shows the dependence of $\Omega^{\prime}(q)$ in a paramagnet for the same parameters as for Fig. 1. It is seen that there is an energy gap and an impulse gap. Figure 2 shows the dependences of $\Omega^{\prime \prime}(q)$ in diamagnet for the previous parameters, except for $a=\pi$. In the region of small $q$, there are fifteen solutions. It can be seen that the two branches have regions of instability. Figures 4-7 show the dependences of the wave numbers $q_{c}$ for which $\Omega^{\prime \prime}\left(q_{c}\right)=0$, on the spin-spin interaction constant $q_{c}(a)$, on the relaxation times $q_{c}\left(\theta \equiv \theta_{v}=\theta_{t}=\theta_{r}\right)$, the spatial dispersion constants $q_{c}\left(\lambda \equiv \lambda_{y m}^{2}=\lambda_{t m}^{2}=\lambda_{r m}^{2}\right)$, the ratio of the velocities $q_{c}\left(\gamma \equiv \gamma_{\eta}=\gamma_{\chi}\right)$ with the same constant parameters. The curves limit the region of wave instability.

Thus, in a locally non-equilibrium continuum with spatial dispersion with an increase in the constants $\lambda_{v m}, \lambda_{t m}$, $\lambda_{r m}$ and $a$ by decreasing $\theta_{v}, \theta_{t}, \theta_{r}$ and $\gamma_{\eta}, \gamma_{\chi}$ the instability region first shrinks and eventually disappears.

The point of occurrence of instability when the parameters determined from the equation $e_{0}\left(q_{c 0}\right)=0$, where $q_{c 0}$ is the wave number corresponding to the maximum 


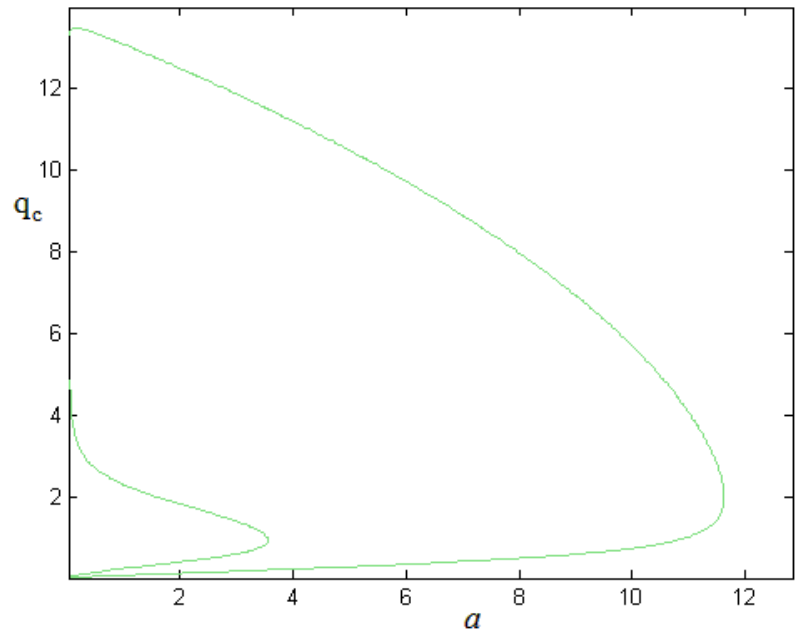

Fig. 4. Dependence of the critical wave number on the spin-spin interaction constant.

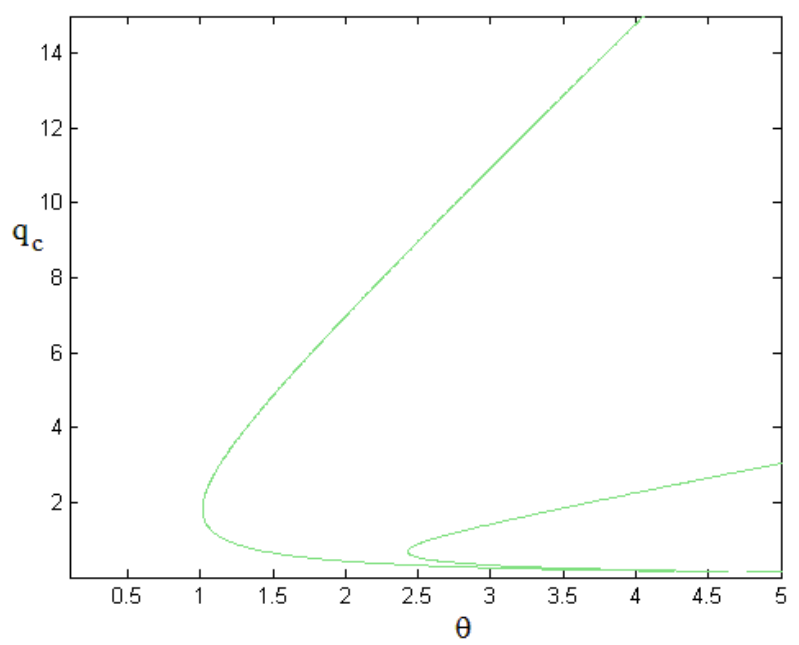

Fig. 5. Dependence of the critical wave number on the relaxation times.

of the imaginary part of the frequency, which is determined from the equation $\left.\left(\partial \Omega^{\prime \prime} / \partial q\right)\right|_{q c 0}=0$. In Figs. $4-7$ the wave number with vertical tangent correspondsto $q_{c 0}$.

Locally equilibrium fluctuations $\left(\tau_{v}, \tau_{t}, \tau_{r} \rightarrow 0\right)$ in the absence of spatial dispersion $\left(\lambda_{v}^{2}, \lambda_{t}^{2}, \lambda_{r}^{2} \rightarrow 0\right)$ on the basis of (11) are determined by the relation

$$
\Omega^{\prime 2}=q^{2}+2 \Omega^{\prime \prime}+3 \Omega^{\prime \prime 2},
$$

in which the imaginary part is determined from equation

$$
\Omega^{\prime \prime 3}+\Omega^{\prime \prime 2}+(1 / 4)\left(1+q^{2}\right) \Omega^{\prime \prime}+(\pi / 2 a) q^{2}=0 .
$$

Since all the coefficients in the second equation are positive, the solutions exist only for $\Omega^{\prime \prime}<0$. Consequently, the locally equilibrium state will be stable. The dependence of $\Omega^{\prime}(q)$ does not have a gap.

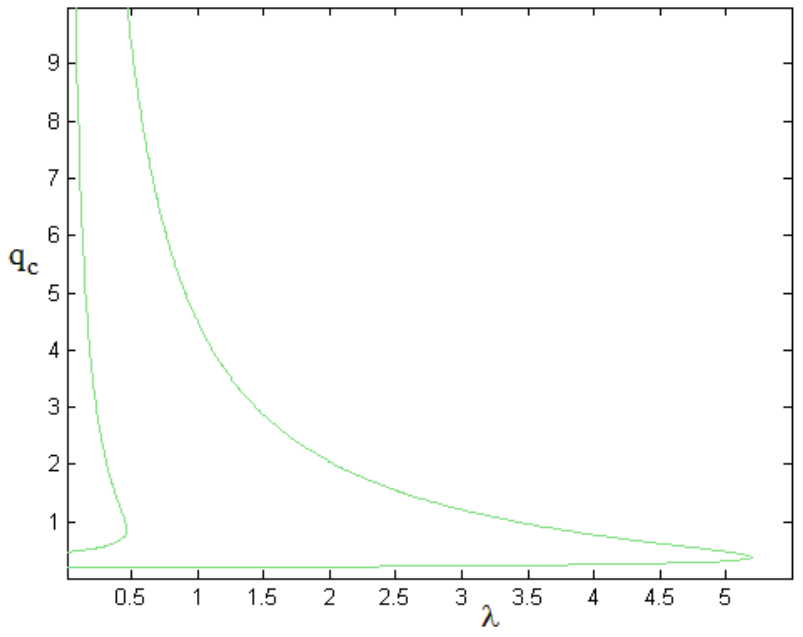

Fig. 6. Dependence of the critical wave number on the spatial dispersion constants.

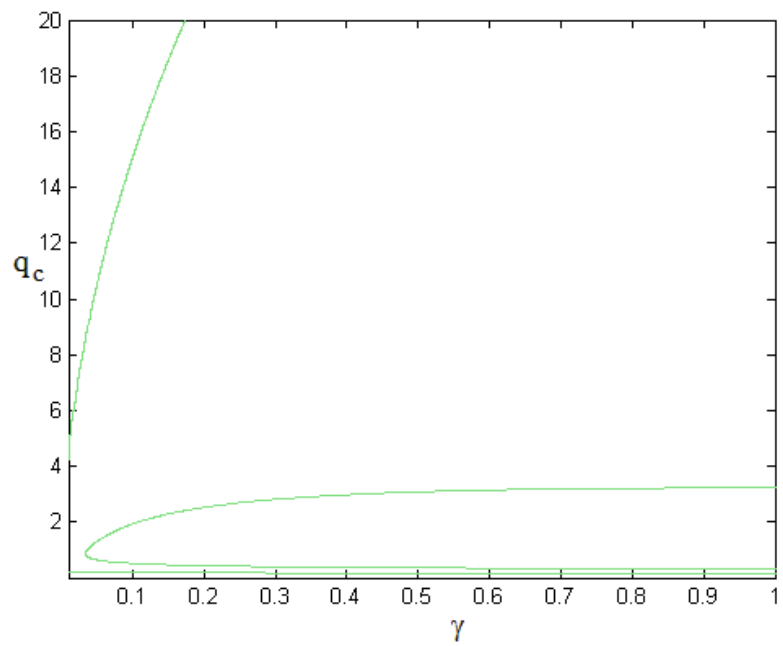

Fig. 7. Dependence of the critical wave number on the ratio of the velocities of the magnetization waves and the speed of light.

\section{Stationary locally non-equilibrium excitations}

Instability is limited to nonlinear effects. Because of the nonlinearity, the effective coupling constants and spatial dispersion increase, and the relaxation times decrease. As a result, as the amplitude increases, the parameters shift to the stability boundary, where the amplitude of the wave is constant. To take into account the nonlinearity, we can assume that the kinetic coefficients in (5) depend on thermodynamic variables, flows, forces and their temporal and spatial derivatives. Next, we restrict ourselves to the dependence of the coefficients on the right-hand sides of Eq. (6) on magnetization and thermodynamic forces.

Consider waves of the form $\boldsymbol{B}, \boldsymbol{M}=\boldsymbol{B}_{0}, \boldsymbol{M}_{0} \exp (i \boldsymbol{k} \boldsymbol{x}-$ $i \omega t)+$ c.c., where $\boldsymbol{B}_{0}, \boldsymbol{M}_{0}$ are complex amplitudes. The linear field Eqs. (8), taking into account the nonlinear in- 
ternal induction $\boldsymbol{B}^{m}=\left(a+a^{\prime} \boldsymbol{M}^{2}\right) \boldsymbol{M}$, give the amplitude ratio at the fundamental frequency $c_{l 1} \boldsymbol{k}(\boldsymbol{k} \boldsymbol{M})+\kappa^{-1} \boldsymbol{M}=$ $\boldsymbol{B}$, where $\kappa=\left(q^{2}-\Omega^{2}\right) /\left(a^{m} \Omega^{2}-a_{\pi}^{m} q^{2}\right), a^{m}=a\left(1+m_{0}^{2}\right)$ is the interaction constant renormalized by the square of the amplitude, $m_{0}^{2}=\left|\boldsymbol{M}_{0}\right|^{2} / M_{a}^{2}$ is the normalized amplitude, $M_{a}^{2}=\left(a / 2 a^{\prime}\right), a^{\prime}>0, a_{\pi}^{m}=a^{m}-4 \pi$, $c_{l 1}=-4 \pi /\left(k^{2}-k_{0}^{2}\right)$. For $a<0$, the region of instability begins with $q=0[5]$ and, consequently, the ground state is ferromagnetic with the magnetization $M_{s}^{2}=-a / 2 a^{\prime}$. We confine ourselves to the cubic nonlinearity in the defining relations. Taking into account the amplitude relation from (1) and (6), the dispersion relation for transverse waves

$$
\left(q^{2}-\Omega^{2}\right)\left(q^{2}-q_{m s}^{2}\right)-4 \pi q_{c s}^{2} q^{2}=0,
$$

where $q_{m s}^{2}=c_{1 s} / c_{0 s}$ and $q_{c s}^{2}=c_{2 s} / c_{0 s}$ is the normalized wave number, the change in the amplitude of stationary waves, $c_{1 s}=i \Omega-\tau_{m} \nu_{m} a^{m} \zeta_{v}^{-1}, c_{2 s}=$ $\tau_{m} \nu_{m} \zeta_{v}^{-1}+\left(q^{2} / a^{m}\right) c_{0 s}, c_{0 s}=a^{m}\left(\theta_{t} \gamma_{\eta n} \zeta_{t}^{-1}+\theta_{r} \gamma_{\chi n} \zeta_{r}^{-1}\right)$, $\zeta_{i}=1+\lambda_{i m}^{2} q^{2}-i \Omega \theta_{i}, i=v, t, r, \nu_{m}=\nu\left(1+\nu_{n} m_{0}^{2}\right)$, $\nu_{n}=m_{a \nu}^{2}\left(1+\nu_{21} \kappa^{-1}+\nu_{31} \kappa^{-2}\right), m_{a \nu}^{2}=M_{a}^{2} / M_{\nu}^{2}$, $M_{\nu}^{2}=\nu / 2 \nu_{1}$ is the effective magnetization, $\nu_{1}, \nu_{21}, \nu_{31}$ are the nonlinear viscosity coefficients in the first Eq. (6), $\gamma_{\eta n}=\gamma_{\eta}\left(1+\eta_{n} m_{0}^{2}\right)$ is the renormalized ratio of shear rates, $\eta_{n}=m_{a \eta}^{2}\left(1+\eta_{21} \kappa^{-1}+\eta_{31} \kappa^{-2}\right), m_{a \eta}^{2}=M_{a}^{2} / M_{\eta}^{2}$, $M_{\eta}^{2}=\eta / \eta_{1}$ is the effective magnetization, $\eta_{1}, \eta_{21}, \eta_{31}$ are nonlinear shear viscosity coefficients in the second equation (6), $\gamma_{\chi n}=\gamma_{\chi}\left(1+\chi_{n} m_{0}^{2}\right)$ is the renormalized ratio of rotational velocities, $\chi_{n}=m_{a \chi}^{2}\left(1+\chi_{21} \kappa^{-1}+\chi_{31} \kappa^{-2}\right)$, $m_{a \chi}^{2}=M_{a}^{2} / M_{\chi}^{2}, M_{\chi}^{2}=\chi / \chi_{1}$ is the effective magnetization, $\chi_{1}, \chi_{21}, \chi_{31}$ are nonlinear rotational viscosity coefficients in the last Eq. (6).

Equation (16) can be reduced to an equation for the frequency in the implicit form

$$
\begin{aligned}
& a_{06} \Omega^{6}+a_{04 n} \Omega^{4}+a_{02 n} \Omega^{2}+a_{00 n} \\
& \quad+\mathrm{i} \Omega\left(a_{05} \Omega^{4}+a_{03 n} \Omega^{2}+a_{01 n}\right)=0,
\end{aligned}
$$

where $a_{06}=f_{\zeta 3}, a_{05}=f_{\zeta 2}, a_{04 n}=-\left(f_{\zeta 1}+f_{\zeta 3} q^{2}+\right.$ $\left.a^{m} f_{\gamma 2}\right), a_{03 n}=-\left(f_{\zeta 0}+f_{\zeta 2} q^{2}+a^{m} f_{\gamma 1}\right), a_{02 n}=f_{\zeta 1} q^{2}+$ $a^{m} f_{\gamma 0}+a_{\pi}^{m} f_{\gamma 2} q^{2}, a_{01 n}=f_{\zeta 0} q^{2}+a_{\pi}^{m} f_{\gamma 1} q^{2}, a_{00 n}=$ $-a_{\pi}^{m} f_{\gamma 0} q^{2}$ are the coefficients, $f_{\zeta 3}=\theta_{v} \theta_{1}, f_{\zeta 2}=$ $\theta_{v} \theta_{t} \zeta_{r \lambda}+\theta_{v} \theta_{r} \zeta_{t \lambda}+\theta_{r} \theta_{t} \zeta_{v \lambda}, f_{\zeta 1}=\theta_{v} \zeta_{t \lambda} \zeta_{r \lambda}+\theta_{t} \zeta_{r \lambda} \zeta_{v \lambda}+$ $\theta_{r} \zeta_{v \lambda} \zeta_{t \lambda}, f_{\zeta 0}=\zeta_{v \lambda} \zeta_{t \lambda} \zeta_{r \lambda}$ are the functions determined by the relaxation times and spatial dispersion, $f_{\gamma 2}=$ $\theta_{1}\left(\tau_{m} \nu_{m}+\theta_{v} \gamma_{0 n} q^{2}\right), f_{\gamma 1}=\tau_{m} \nu_{m}\left(\theta_{t} \zeta_{r \lambda}+\theta_{r} \zeta_{t \lambda}\right)+\left(\theta_{v} \gamma_{1 n}+\right.$ $\left.\theta_{1} \zeta_{v \lambda} \gamma_{0 n}\right) q^{2}, f_{\gamma 0}=\tau_{m} \nu_{m} \zeta_{t \lambda} \zeta_{r \lambda}+\zeta_{v \lambda} \gamma_{1 n} q^{2}$ are the functions additionally determined by linear and nonlinear viscosities, $\gamma_{0 n}=\gamma_{\eta n}+\gamma_{\chi n}, \gamma_{1 n}=\theta_{t} \zeta_{r \lambda} \gamma_{\eta n}+\theta_{r} \zeta_{t \lambda} \gamma_{\chi n}$. For $m_{0}^{2} \rightarrow 0$, Eq. (17) is transformed into (11).

Since the "viscosities" depend on the frequency, in an explicit form, Eq. (17) is an equation of the tenth power with respect to frequency. In the weak dispersion approximation of nonlinear viscosities $\left|\delta_{21} \kappa^{-1}+\delta_{31} \kappa^{-2}\right|<<1$, $\delta=\nu, \eta, \chi$ the coefficients will be determined by the relations $\nu_{m}=\nu\left(1+m_{a \nu}^{2} m_{0}^{2}\right), \gamma_{\eta n}=\gamma_{\eta}\left(1+m_{a \eta}^{2} m_{0}^{2}\right)$, $\gamma_{\chi n}=\gamma_{\chi}\left(1+m_{a \chi}^{2} m_{0}^{2}\right)$. Since in this approximation the coefficients do not depend on the frequency, Eq. (17) reduces to (11) by replacing $\nu, \gamma_{\eta}, \gamma_{\chi} \rightarrow \nu_{m}, \gamma_{\eta n}, \gamma_{\chi n}$.
Consequently, formulae (11)-(15) will describe nonlinear excitations with a constant amplitude for the indicated substitution. From the equation $e_{0}\left(q, m_{0}^{2}\right)=0$ one can find the amplitude $m_{0}^{2}(q)$ and substituting it in (13) for $\Omega^{\prime \prime}=0$, we obtain the dispersion relation of waves of constant amplitude.

The equation $e_{0}\left(q^{2}, m_{0}^{2}\right)=0$ can be written relative to the square of the amplitude

$$
\Sigma_{n=0}^{n=8} e_{0 n} m_{0}^{2 n}=0
$$

where $e_{0 n}=\Sigma_{m, l=0}^{m+l=n}\left(i_{00 m} I_{00 l}+i_{10 m} I_{10 l}\right)+a_{06}^{2} I_{20 n}$ are coefficients depending on $q^{2}, 0 \leq l, m \leq 4, i_{00 m}=$ $\Sigma_{k, r=0}^{k+r=m}\left(a_{00 k} a_{03 r}-a_{02 k} a_{01 r}\right), I_{00 l}=a_{05}\left(2 a_{06} a_{01 k}-\right.$ $\left.a_{05} a_{02 k}\right) \delta_{k l}+\sum_{k, r=0}^{k+r=l}\left(a_{05} a_{04 k} a_{03 r}-a_{06} a_{03 k} a_{03 r}\right), i_{10 m}=$ $a_{05} a_{00 k} \delta_{k m}-\sum_{k, r=0}^{k+r=m} a_{04 k} a_{01 r}, \quad I_{10 l}=a_{05} i_{1 l}+$ $a_{06} \sum_{k, r=0}^{k+r=l} a_{03 k} a_{01 r}, \quad I_{20 n}=\sum_{k, r, p=0}^{k+r+p=n} a_{01 k} a_{01 r} a_{01 p}$ are the terms of the coefficients, $0 \leq k, r, p \leq 2, \delta_{k l}$ is the delta symbol, $a_{000}=-a_{\pi} f_{\gamma 00} q^{2}, a_{001}=-\left(a f_{\gamma 00}+\right.$ $\left.a_{\pi} f_{\gamma 01}\right) q^{2}, a_{002}=-a f_{\gamma 01} q^{2}, a_{010}=\left(f_{\gamma 00}+a_{\pi} f_{\gamma 10}\right) q^{2}$, $a_{011}=\left(f_{\gamma 01}+a f_{\gamma 10}+a_{\pi} f_{\gamma 11}\right) q^{2}, \quad a_{012}=a f_{\gamma 11} q^{2}$, $a_{020}=a f_{\gamma 00}+\left(f_{\zeta 1}+a_{\pi} f_{\gamma 20}\right) q^{2}, a_{021}=a\left(f_{\gamma 00}+f_{\gamma 01}\right)+$ $\left(a f_{\gamma 20}+a_{\pi} f_{\gamma 21}\right) q^{2}, a_{022}=a\left(f_{\gamma 01}+f_{\gamma 21} q^{2}\right), a_{030}=-f_{\zeta 0}-$ $f_{\zeta 2} q^{2}-a f_{\gamma 10}, a_{031}=-a\left(f_{\gamma 10}+f_{\gamma 11}\right), a_{032}=-a f_{\gamma 11}$, $a_{040}=-f_{\zeta 1}-f_{\zeta 3} q^{2}-a f_{\gamma 20}, a_{041}=-a\left(f_{\gamma 20}+f_{\gamma 21}\right)$, $a_{042}=-a f_{\gamma 21}$ are the coefficients for different degrees of the square of the amplitude, $f_{\gamma 00}=a^{-1} \zeta_{t \lambda} \zeta_{r \lambda}+\gamma_{1 n 0} \zeta_{v \lambda}$, $f_{\gamma 10}=a^{-1}\left(\theta_{t} \zeta_{r \lambda}+\theta_{r} \zeta_{t \lambda}\right)+\left(\theta_{v} \gamma_{1 n 0}+\theta_{1} \zeta_{v \lambda} \gamma_{0}\right) q^{2}, f_{\gamma 20}=$ $\theta_{1}\left(a^{-1}+\theta_{v} \gamma_{0} q^{2}\right)$ and $f_{\gamma 01}=a^{-1} \zeta_{t \lambda} \zeta_{r \lambda} m_{a \nu}^{2}+\gamma_{1 n m} \zeta_{v \lambda}$, $f_{\gamma 11}=a^{-1}\left(\theta_{t} \zeta_{r \lambda}+\theta_{r} \zeta_{t \lambda}\right) m_{a \nu}^{2}+\left(\theta_{v} \gamma_{1 n m}+\theta_{1} \zeta_{v \lambda} \gamma_{0 m}\right) q^{2}$, $f_{\gamma 21}=\theta_{1}\left(a^{-1} m_{a \nu}^{2}+\theta_{v} \gamma_{0 m} q^{2}\right)$ are functions determined by linear and nonlinear viscosities, $\gamma_{1 n 0}=\theta_{t} \zeta_{r \lambda} \gamma_{\eta}+$ $\theta_{r} \zeta_{t \lambda} \gamma_{\chi}, \gamma_{0 m}=\gamma_{\eta} m_{a \eta}^{2}+\gamma_{\chi} m_{a \chi}^{2}, \gamma_{1 n m}=\theta_{t} \zeta_{r \lambda} \gamma_{\eta} m_{a \eta}^{2}+$ $\theta_{r} \zeta_{t \lambda} \gamma_{\chi} m_{a \chi}^{2}$ are linear and non-linear ratio of velocities. The numerical solution of (18) is shown in Fig. 8 with the previous parameters and $m_{a \delta}^{2}=0.01$. The amplitude interval coincides with the instability interval (positive imaginary frequencies). With increasing $m_{a \delta}^{2}$, the dependence of $m_{0}^{2}(q)$ becomes ambiguous near the upper boundary of the instability.

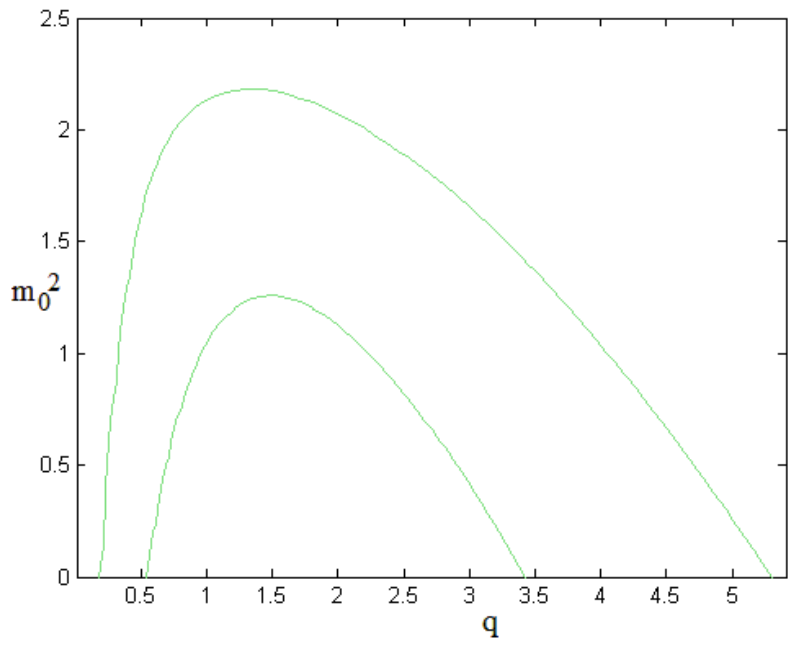

Fig. 8. Dependence of the square of the amplitude of stationary waves on the wave number. 
The wave number $q_{c 0}$ corresponding to the maximum amplitude is determined from the equation $\left(\partial m_{0}^{2} / \partial q^{2}\right)_{q c 0}=0$. The critical parameters are related by the equation $e_{00}\left(q_{c 0}\right)=0$. Below the critical point, the stationary amplitude is zero and above the critical point the stationary amplitude is finite. Hence the maximum stationary amplitude can be taken as the order parameter and consider the transition through the instability point as a phase transition. In a new phase there exist both damped (normal) excitations and undamped excitations. Wave packets from the instability region propagate without damping, as a result of scattering, a decrease in the amplitude of the packet waves causes an instability that restores the stationary wave amplitudes (analogous to superfluidity, superconductivity).

\section{Locally non-equilibrium standing waves}

Consider harmonic oscillations in a bounded volume in the form of a rectangular parallelepiped with sides $l_{1}, l_{2}$, $l_{3}$. Suppose that there are no singularities of the fields and vanish on the boundary of the field. Then the solution of Eqs. (7), (8) can be sought in the form

$$
\begin{aligned}
& F_{x}=F_{1}\left[\cos k_{1} x \sin k_{2} y \sin k_{3} z\right] \exp (-\mathrm{i} \omega t)+\text { c.c. }, \\
& F_{y}=F_{2}\left[\sin k_{1} x \cos k_{2} y \sin k_{3} z\right] \exp (-\mathrm{i} \omega t)+\text { c.c. }, \\
& F_{z}=F_{3}\left[\sin k_{1} x \sin k_{2} y \cos k_{3} z\right] \exp (-\mathrm{i} \omega t)+\text { c.c., }
\end{aligned}
$$
where $\boldsymbol{F}=\boldsymbol{B}, \boldsymbol{M}, k_{1}=\left(\pi / l_{1}\right) n, k_{2}=\left(\pi / l_{2}\right) m, k_{3}=$ $\left(\pi / l_{3}\right) p, n, m, p=0,1, \ldots$ are integers. The components of induction and magnetization are related by the relations $F_{n} k_{n}=0$. Using the amplitude relation from (1) and (6), the dispersion relation for standing waves

$$
\left(q_{\nu}^{2}-\Omega^{2}\right)\left(q_{\nu}^{2}-q_{m s}^{2}\right)-4 \pi q_{c s}^{2} q_{\nu}^{2}=0,
$$

where $q_{\nu}^{2} \equiv \lambda_{m}^{2} k_{n m p}^{2}, k_{n m p}^{2}=k_{1}^{2}+k_{2}^{2}+k_{3}^{2}$ is the discrete wave number. Equation (20) is transformed into (16) by replacing the discrete wave number by the continuous wave number $q_{\nu}^{2} \rightarrow q^{2}$. Consequently, the dispersion and attenuation of standing waves of small amplitude will be determined by the system of Eqs. (13), (15) with $q^{2} \rightarrow q_{\nu}^{2}$ replaced. In the region of instability, standing stationary waves with a wave number $q_{\nu}^{2}$ and amplitude of (18) will exist.

\section{Propagation of waves in locally non-equilibrium continuum with spatial dispersion}

We consider the excitation of waves by an external source on the boundary of the medium. In this case, the frequency will be real $\left(\Omega^{\prime} \equiv \Omega, \Omega^{\prime \prime}=0\right)$ and the wave vector $\boldsymbol{q}=\boldsymbol{q}^{\prime}+\mathrm{i} \boldsymbol{q}^{\prime \prime}$ complex. Equation (11) is written with respect to the square of the wave number

$$
b_{08} q^{8}+b_{06} q^{6}+b_{04} q^{4}+b_{02} q^{2}+b_{00}=0,
$$

where $b_{08}=b_{08}^{\prime}+\mathrm{i} b_{08}^{\prime \prime}, b_{08}^{\prime}=a_{004}, b_{08}^{\prime \prime}=\Omega a_{014}$, $b_{06}=b_{06}^{\prime}+\mathrm{i} b_{06}^{\prime \prime}, b_{06}^{\prime}=a_{003}+\Omega^{2} a_{023}, b_{06}^{\prime \prime}=\Omega\left(a_{013}+\right.$ $\left.\Omega^{2} a_{033}\right), b_{04}=b_{04}^{\prime}+\mathrm{i} b_{04}^{\prime \prime}, b_{04}^{\prime}=a_{002}+\Omega^{2} a_{022}+\Omega^{4} a_{042}$, $b_{04}^{\prime \prime}=\Omega\left(a_{012}+\Omega^{2} a_{032}\right), b_{02}=b_{02}^{\prime}+\mathrm{i} b_{02}^{\prime \prime}, b_{02}^{\prime}=a_{001}+$ $\Omega^{2} a_{021}+\Omega^{4} a_{041}, b_{02}^{\prime \prime}=\Omega\left(a_{011}+\Omega^{2} a_{031}+\Omega^{4} a_{051}\right)$, $b_{00}=b_{00}^{\prime}+\mathrm{i} b b_{00}^{\prime \prime}, b_{00}^{\prime}=\Omega^{2} a_{020}+\Omega^{4} a_{040}+\Omega^{6} a_{06}$, $b_{00}^{\prime \prime}=\Omega\left(\Omega^{2} a_{030}+\Omega^{4} a_{050}\right)$ are the coefficients. In the general case, the solutions $q_{n}^{2}(21)$ are expressed in terms of the square roots of the cubic equation (the resolvent of the original equation). The polarization of the waves is determined by (9), in which the wave numbers $q_{n}^{2}$. The real and imaginary parts of the wave number

$$
q_{n}^{\prime \prime \prime \prime}=\left(\frac{1}{2}\right)^{\frac{1}{2}}\left[\left(\left(q_{n}^{2 \prime}\right)^{2}+\left(q_{n}^{2 \prime \prime}\right)^{2}\right)^{\frac{1}{2}} \pm q_{n}^{2 \prime}\right]^{\frac{1}{2}},
$$
where $q^{2 \prime}=\boldsymbol{q}^{\prime 2}-\boldsymbol{q}^{\prime \prime 2}$ and $q^{2 \prime \prime}=2 \boldsymbol{q}^{\prime} \boldsymbol{q}^{\prime \prime}$ are the real and imaginary parts of the square of the wave number.

It follows from (22) that there exist frequencies $\Omega_{l}$ satisfying the equation $q_{n}^{2 \prime}\left(\Omega_{l}\right)=0$. These boundary frequencies separate the transmission regions $q_{n}^{2 \prime}>0\left(q_{n}^{\prime}>\right.$ $\left.q_{n}^{\prime \prime}\right)$ and the non-transmission $q_{n}^{2 \prime}<0\left(q_{n}^{\prime}<q_{n}^{\prime \prime}\right)$. At the points $\Omega_{l}$, the wave numbers $q_{n l}^{\prime}=q_{n l}^{\prime \prime}=2^{-\frac{1}{2}}\left|q_{n}^{2 \prime \prime}\left(\Omega_{l}\right)\right|^{\frac{1}{2}}$. There exist critical frequencies $\Omega_{c}$ satisfying the equation $q_{n}^{2 \prime \prime}\left(\Omega_{c}\right)=0$. In the transmission region $q_{n}^{2 \prime}\left(\Omega_{c}\right)>0$ at the transmission frequencies $q_{n c}^{\prime}=\left(q_{n}^{2 \prime}\left(\Omega_{c}\right)\right)^{\frac{1}{2}}, q_{n c}^{\prime \prime}=0$ the waves propagate without damping. In the nontransmission region $q_{n}^{2 \prime}\left(\Omega_{c}\right)<0$, the waves do not penetrate into the medium at the opacity frequencies $q_{n c}^{\prime}=0$, $q_{n c}^{\prime \prime}=\left(-q_{n}^{2 \prime}\left(\Omega_{c}\right)\right)^{\frac{1}{2}}$

The spatial dispersion constants determine the first two coefficients in (21) and change the remaining coefficients. Hence, spatial dispersion creates additional modes and shifts the characteristic frequencies and asymptotic velocities of the waves.

For a weak spatial dispersion and a small ratio of the velocities in the coefficients (22), one can ignore terms of order $\lambda^{6}, \lambda^{4}$ and $\gamma \lambda^{2}$. Then Eq. (21) reduces to a seconddegree equation, the solution of which gives the SE and ES branches $q_{n}^{2}=\left(\frac{1}{2} b_{04}\right)\left(-b_{02} \pm d^{\frac{1}{2}}\right)$, where $n=1,2$, $d=b_{02}^{2}-4 b_{04} b_{00}$. The real and imaginary parts of the discriminant $d^{\prime}=b_{02}^{\prime 2}-b_{02}^{\prime \prime 2}-4\left(b_{04}^{\prime} b_{00}^{\prime}-b_{04}^{\prime \prime} b_{00}^{\prime \prime}\right)$ and $d^{\prime \prime}=2 b_{02}^{\prime} b_{02}^{\prime \prime}-4\left(b_{04}^{\prime} b_{00}^{\prime \prime}+b_{04}^{\prime \prime} b_{00}^{\prime}\right)$ and the root of the discriminant $(\sqrt{d})^{\prime, \prime \prime}=\sqrt{|d| \pm d^{\prime}} / \sqrt{2}$. As a result, the real and imaginary parts of the square of the wave number

$$
\begin{aligned}
& q_{n}^{2 \prime}=\left[-b_{04}^{\prime} b_{02}^{\prime}-b_{04}^{\prime \prime} b_{02}^{\prime \prime}\right. \\
& \left. \pm\left(b_{04}^{\prime}\left(d^{\frac{1}{2}}\right)^{\prime}+b_{04}^{\prime \prime}\left(d^{\frac{1}{2}}\right)^{\prime \prime}\right)\right] / 2\left|b_{04}\right|^{2}, \\
& q_{n}^{2 \prime \prime}=\left[-b_{04}^{\prime} b_{02}^{\prime \prime}+b_{04}^{\prime \prime} b_{02}^{\prime}\right. \\
& \left.\quad \pm\left(b_{04}^{\prime}\left(d^{\frac{1}{2}}\right)^{\prime \prime}-b_{04}^{\prime \prime}\left(d^{\frac{1}{2}}\right)^{\prime}\right)\right] / 2\left|b_{04}\right|^{2} .
\end{aligned}
$$

Figure 9 shows the dependences $q^{\prime}(\Omega), q^{\prime \prime}(\Omega)$ on the basis of $(22),(23)$ in the paramagnet for the parameters $\theta_{v}=4, \theta_{t}=2, \theta_{r}=4, \gamma_{\eta}=0.05, \gamma_{\chi}=0.07, a=10 \pi$, $\lambda_{y m}^{2}=\lambda_{t m}^{2}=\lambda_{r m}^{2}=0.04$. It can be seen that in the high-frequency region the SE wave has two transparency frequencies and a cut-off frequency, the ES wave has two transparency frequencies. In addition, in the low-frequency region, the SE has a transparency frequency, the ES wave has two frequencies of opacity and boundary frequency. Figure 10 shows the dependences 
of $q^{\prime}(\Omega), \mathrm{q}^{\prime \prime}(\Omega)$ in a diamagnet for the previous parameters, except for $a=\pi$. In the high-frequency region, the SE has an opacity frequency, the ES has a frequency of transparency, and in the low-frequency region the SE has an opacity frequency, the ES have an opacity frequency and a cut-off frequency. The SE wave does not propagate in the diamagnet, for $\Omega \rightarrow 0$ the $q^{\prime}(\Omega)$ and $q^{\prime \prime}(\Omega)$ dependences merge (the skin effect).

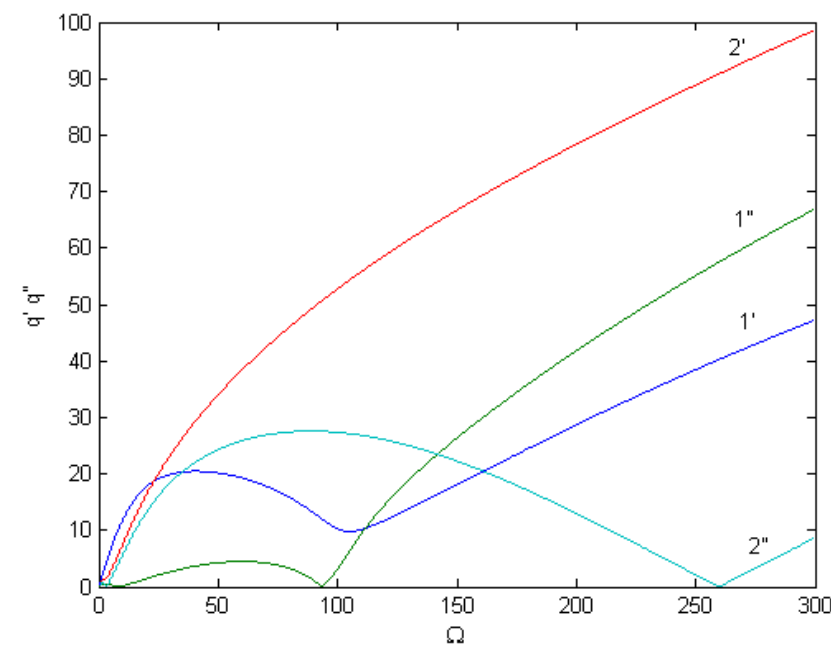

Fig. 9. Dependence of the real $\left(1^{\prime}, 2^{\prime}\right)$ and imaginary $\left(1^{\prime \prime}, 2^{\prime \prime}\right)$ parts of the wave number on the frequency for paramagnets.

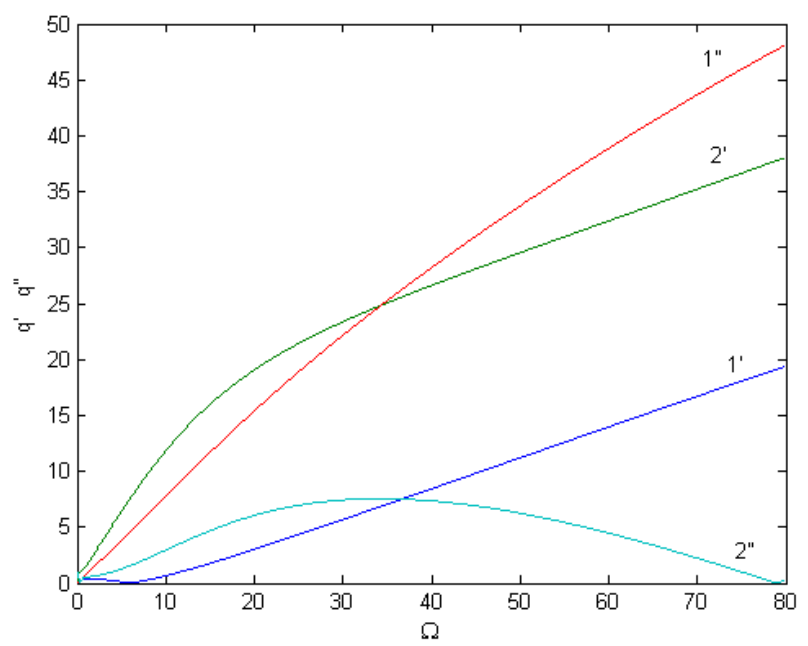

Fig. 10. Dependence of the real $\left(1^{\prime}, 2^{\prime}\right)$ and imaginary $\left(1^{\prime \prime}, 2^{\prime \prime}\right)$ parts of the wave number on the frequency for diamagnets.

The frequencies of transparency and opacity can be explained as follows. Electromagnetic (EM) wave excites oscillations of magnetization with a period less than the relaxation time of magnetization. The variable magnetization emits an induced wave in a phase with EM wave at a transparency frequency. As a result, the energy of the EM wave is preserved. The propagation of EM wave at the frequency of transparency is analogous to the effect of self-induced transparency in optics [15]. In the latter, a short pulse of coherent light excites atoms at a resonance frequency in a time less than the relaxation time of polarization and then stimulates the atoms to stimulated emission, and turns the energy into a pulse. At the opacity frequency, the magnetization radiates in antiphase with the EM wave, consequently, the EM wave decays at the wavelength.

In the locally equilibrium state $\left(\tau_{v}, \tau_{t}, \tau_{r} \rightarrow 0\right)$ without spatial dispersion $\left(\lambda_{v}^{2}, \lambda_{t}^{2}, \lambda_{r}^{2} \rightarrow 0\right)$, Eq. (21) gives the ES wave with

$$
\begin{aligned}
& q^{\prime}=\Omega\left(1+\left(1+\Omega_{\alpha}^{2}\right)^{\frac{1}{2}}\right)^{\frac{1}{2}} / 2^{\frac{1}{2}}, \\
& q^{\prime \prime}=\Omega^{2}(1-\alpha) / 2^{\frac{1}{2}}\left(\alpha^{2}+\Omega^{2}\right)\left(1+\left(1+\Omega_{\alpha}^{2}\right)^{\frac{1}{2}}\right)^{\frac{1}{2}},
\end{aligned}
$$

where $\Omega_{\alpha}^{2}=\Omega^{2}(1-\alpha)^{2} /\left(\alpha^{2}+\Omega^{2}\right)^{2}$. It is clear that in the local equilibrium state the characteristic frequencies $\Omega_{l}, \Omega_{c}$ are absent.

\section{The discussion of the results}

In the above discussion, stationary waves were not investigated with respect to the amplitude instability. The development of modulation instability leads to the formation of solitary envelope waves (solitons). In solitary waves, the spreading of a wave packet due to dispersion is compensated by its contraction due to nonlinearity. In the region of instability, the solitons will be stationary. The phase with solitons will be more ordered than the phase with wave packets.

A non-equilibrium state can arise in a limited region due, for example, to a local change in temperature, a constant, or relaxation times. This region of a nonequilibrium continuum can further change (contract, collapse, expand) or have stable dimensions (for example, spontaneous stable thermoelectromagnetic solitons).

We have considered above the dynamics of magnetization at a constant temperature. Taking into account the change in temperature in space and time gives additional effects similar to those in a liquid with internal rotation [9]. In particular, a thermomagnetic effect and the propagation of thermomagnetic waves without damping are possible.

By analogy, we can write the equations of motion of polarization, magnetization, current for a locally nonequilibrium multicomponent continuum, in particular an antiferromagnet.

Calculations showed that in the liquid there exist unstable coupled transverse waves of momentum and spin (internal rotation) for long relaxation times and weak spatial dispersion, as above, waves of the magnetic vector potential and magnetization were considered.

\section{Acknowledgments}

The author is grateful to F.V. Lisovskii for discussing the work. 


\section{References}

[1] F. Bloch, Zeitschr. Phys. 61, 206 (1930).

[2] L. Landau, E. Lifshits, Phys. Zs. Sowjet. 8, 153 (1935).

[3] F. Bloch, Phys. Rev. 70, 460 (1946).

[4] A.I. Akhiezer, V.G. Bar'yakhtar, S.V. Peletminskii, Spin Waves, North-Holland, New York 1968.

[5] A.F. Kabychenkov, Acta Phys. Pol. A 123, 695 (2013).

[6] A.F. Kabychenkov, in: Proc. XXII Int. Conf. "New in Magnetism and Magnetic Materials", Astrakhan, Astrakhan State University, Astrakhan 2012, p. 42 (in Russian).

[7] S.R. de Groot, P. Mazur, Non-Equilibrium Thermodynamics, North-Holland, Amsterdam 1962.
[8] I. Gyarmati, Non-Equilibrium Thermodynamics, Springer-Verlag, Berlin 1970.

[9] A.F. Kabychenkov, Techn. Phys. 54, 1116 (2009).

[10] I.P. Bazarov, Thermodynamics, Higher School, Moscow 1991.

[11] P.A. Sturrock, J. Appl. Phys. 31, 2052 (1960).

[12] J.R. Pierce, Almost All about Waves, MIT Press, Cambridge (Mass.) 1974

[13] M.V. Nezlin, Phys. Usp. 19, 946 (1976).

[14] I.E. Tamm, Phys. Usp. 68, 387 (1959) (in Russian). DOI:

[15] P.G. Kryukov, V.S. Letokhov, Sov. Phys. Usp. 12, $641(1970)$. 